\title{
Technik, Digitalität und Raum - Konzeptionelle Überlegungen zu den Geographien alltäglichen Technikgebrauchs
}

\author{
T. Felgenhauer \\ Institut für Geographie, Friedrich-Schiller-Universität Jena, Germany \\ Correspondence to: T. Felgenhauer (tilo.felgenhauer@uni-jena.de)
}

Received: 2 May 2014 - Revised: 28 August 2014 - Accepted: 1 September 2014 - Published: 8 April 2015

Kurzfassung. The article reviews the relationship between technology and geography from a social science perspective. Especially, the everyday use of technology is discussed as a form of "geography-making". Making use of technology means that an interaction between lay users and experts (the designer of an abstract system and its user interface) takes place which basically implies the use and translation of differing spatial symbolisations: mainly numerical/algorithmic, deictic, and toponymical terms.

\section{Einleitung - Technik, Raum und die Geographie}

Nähert man sich den Begriffen Technik und Raum, scheint es fast ebenso schwierig ihren genauen Geltungsbereich zu beschreiben wie es schwer fällt, Wirklichkeitsbereiche zu finden, die von ihnen ausgespart bzw. unbezeichnet blieben. Technik und Raum bilden zwei theoretisch und empirisch schwierige Globalkategorien, die wohl überall relevant und gleichwohl nirgends in Reinform anzutreffen sind. Schon deshalb muss eine notwendig perspektivische Heuristik das Thema aufschlüsseln helfen. Aus der Sicht des kritischen Humangeographen ließen sich vorläufig vielleicht drei Aspekte der Verknüpfung des Technischen mit dem Räumlichen identifizieren. Erstens wäre Technik als Mittel wissenschaftlich-geographischen Arbeitens aufzufassen. Verstanden als Mittelverwendung ist das Technische so alt wie die geographische Forschung selbst - von den Anfängen der Kartographie über genuin geographische Techniken der Beobachtung bis hin zur digitalen Hochtechnologie im Bereich GIS und Fernerkundung. Zweitens könnte Technik als Thema der theoretischen und methodischen geographischen Reflexion behandelt werden. Denn Geographen verwenden technische Mittel nicht nur, sie fragen auch in zunehmend kritisch-reflexiver Haltung, welche technischen Instrumente welche geographische Wirklichkeit erzeugen. Drittens kann die Geographin beobachten, dass Technik nicht nur ein Mittel und Thema des wissenschaftlichen Arbeitens bildet, son- dern eine wichtige Facette alltäglichen Geographie-Machens darstellt - der Geograph tritt dann als Beobachter und Rekonstrukteur technisch geprägter Lebenswelten in Erscheinung. Um diesen letzten Punkt, der alltagsweltlichen Konstruktion technischer Geographien, soll es im Folgenden gehen - mit dem Schwerpunkt auf digitalen Techniken.

Sozialgeographische Zugänge adressieren das Technische als zentralen Teilaspekt der großen Umbrüche der Moderne. Technische Innovationen sind gleichzeitig Grundlage und Ausdruck ,gesellschaftlicher Raumverhältnisse“ (Werlen, 210:321ff), die sich im Wandel befinden. Vor allem Mittel der Mobilität und Kommunikation bilden wichtige Instanzen der Neustrukturierung des Gesellschaft-RaumVerhältnisses. Aktuell stellt sich die Frage nach der technischen Dimension des Alltagshandelns unter den gegenwärtigen Bedingungen der Digitalisierung gleichzeitig auf vertraute und auf neue Weise: Einerseits werden digitale Medien und Technologien als „Vollender“ der Globalisierung, d.h., als letztes Glied in der Reihe der Mittel zur Raumüberwindung gesehen. Andererseits wird mit der Digitalisierung bereits eine grundlegend neue Geographie technisierter Lebenswelten verbunden, die sich nicht durch eine Steigerung bekannter Raumüberwindungsfiguren auszeichnet, sondern durch ein Verlassen der bekannten alltäglichen Handlungs- und wissenschaftlichen Repräsentationslogiken insgesamt. Vor dem Hintergrund solcher Szenarien geht es im Folgenden um Überlegungen zur empirischen Untersu- 
chung des „Geographie-Machens“ (Werlen, 1998, m. Bez. auf Hartke) durch das alltägliche, routinenhafte Interagieren mit technischen Medien und Artefakten.

Der folgende zweite Abschnitt widmet sich der Darstellung, Kritik und Weiterentwicklung raumzentrierter Ansätze, welche primär die Kartierung von Technik als Objekt im Raum zum Gegenstand haben. Damit ist, wie zu zeigen sein wird, eine Tendenz zur Reduktion des Technischen auf „Hardware“ verbunden. Der dritte Teil diskutiert Theorien und Modelle, welche die räumliche Dimension der technisierten Moderne in den Blick nehmen. Konzepte der ,raumzeitlichen Entankerung“ (Werlen, 2010, 29ff), der „Spaces of Flow“ (Castells, 2000 [1996]), des ,coded space/code space" (Dodge und Kitchin, 2005a; 2011, 16ff), und der multidimensionalen, nicht-statischen Verknüpfung von Ort und Technik (Thrift und French, 2002; Graham, 1998, 2005) werden kritisch auf ihr Erklärungspotential hin geprüft. Der vierte Abschnitt untersucht die Tauglichkeit des Begriffes der Digitalisierung für die Neubeschreibung des Technik-RaumVerhältnisses im Hinblick auf die Modellierung des alltäglichen Interagierens mit technischen Artefakten. Hier wird das Argument entwickelt, dass der Begriff „Digitalisierung“ zwar die Binnenfunktion technischer Systeme zu fassen vermag, aber die Begegnungsweisen des Laien mit dem Technischen nicht deutlich genug adressiert. Der fünfte Teil soll das Interaktionshandeln mit Technik als Form der Kommunikation zwischen Laien und Expertensystemen konzeptualisieren. Auf dieser Grundlage soll nach dem raumbezogenen Bedeutungsgehalt technischer Praxis gefragt werden.

\section{Die Verortung des Technischen im Raum}

Mit der Entwicklung und Anwendung kartographischer Techniken beginnt die Operation des Verortens und Ordnens materieller Objekte. Dieser klassische Zugriff auf das Räumliche hat historisch immer auch die Erfassung technischer Objekte eingeschlossen: mit Hilfe von Technik wurde (und wird) Technik kartiert. Nach diesem Prinzip hat etwa die klassische Wirtschafts- und Transportgeographie das Technische als materielle Struktur erfasst, was man heute mit dem Begriff „Hardware“ bezeichnen könnte. Verkehrswege, Güterströme, Infrastrukturen und Architekturen erschienen als Arrangements materieller, technischer Artefakte. So hat etwa Horvath (1974) - in einer heute fast klassisch zu nennenden Studie - am Beispiel der Stadt Lansing (Michigan) gezeigt, dass das Automobil eine überwältigende Dominanz in der Flächennutzung erlangt hat. Indem er Straßen, Parkflächen, Garagen und Wartungs- und Infrastruktureinrichtungen kartographisch ausweist, zeigt sich der raumprägende Charakter dieser Technologie. Horvath prägt in diesem $\mathrm{Zu}-$ sammenhang den Ausdruck des „machine space“. Mit dieser Studie präsentierte Horvath ein beeindruckendes Symptom der technischen Überprägung der Erdoberfläche in der klassischen Moderne. Die Studie weist ein hohes kritisches
Potential auf. Die systematische Fokussierung materieller und gebauter Wirklichkeit hat den Blick für die offenkundigen Transformationen der Erdoberfläche, wie sie die Moderne mit sich brachte, sensibilisiert. Sie steht aber gleichzeitig auch für eine Beschränkung auf die „Hardware“-Seite des Technischen. Technik erscheint abgelöst von subjektiven und sozialen Sinngebungen. Sie tritt vor allem als räumlicher Ausdruck, aber nicht eigentlich als Medium des menschlichen/sozialen Handelns auf. Dass diese Beschränkung sowohl für klassische wie auch für einen großen Teil der aktuellen Studien der Geographien des Technischen gilt, möchte ich mit Blick auf einige jüngere Beispiele illustrieren.

Aktuell bilden digitale Technologien den Schwerpunkt humangeographischer Betrachtungen (s. stellv.: Graham, 1998; Dodge und Kitchin, 2005a, b, 2011; Thrift und French, 2002; Thrift, 2004; Tranos, 2013). Im Sinne ihrer Verbreitung im Raum untersucht etwa Torrens (2008) die Versorgung der Stadt Salt Lake City mit drahtlosen Internetzugangsmöglichkeiten. Er kartiert die Dichte von Zugangspunkten für jeden Ort im Stadtgebiet. Diese Versorgungsdichte wird kartographisch als dritte Dimension der zweidimensionalen „Unterlage" der Karte des Stadtgebietes hinzugefügt. Damit hat sich ein neues empirisches Themenfeld für die Geographie erschlossen. Dieses wird aber im Prinzip mit derselben räumlich-materialisierenden Logik erschlossen wie es die Studie von Horvath zu Beginn der 1970er Jahre mit Bezug auf die Automobiltechnik bereits tat. Ein lediglich abstrakt messbares Phänomen wird durch dessen kartographische Repräsentation reifiziert ${ }^{1}$.

Die Studie von Licoppe et al. (2008) zur individuellen Handy-Nutzung im Alltag steht stellvertretend für eine Erweiterung dieser klassischen ,verräumlichenden“ Herangehensweise. Ihr Fokus besteht in der Verortung, Nachzeichnung und Kartierung der individuellen Nutzungsmomente. Wo welches Untersuchungssubjekt das Handy benutzt hat, wird mit einem Punkt auf der Karte vermerkt. Zusammen mit Informationen über den jeweiligen Zeitpunkt des Gesprächs lassen raumzeitliche Muster der Nutzung erkennen, die entsprechend mit der körperlich-physischen Mobilität der Nutzer korreliert werden - ganz ähnlich der Herangehensweise der Zeitgeographie. Der Fernpendler beispielsweise unterscheidet sich bei der Nutzungsintensität und den Orten des Gebrauchs erwartungsgemäß von der urbanen Vieltelefoniererin. Damit wird ein Schritt in Richtung Handlungsund Alltagszentrierung vollzogen, was aber noch nicht die Perspektive aus Nutzersicht abzubilden vermag. Der kartographische Blick erzeugt seine eigene, dem Geographen so vertraute Geographie der „Draufsicht“ auf das Geschehen.

Diese (und viele andere) Beispiele illustrieren, wie wenig letztlich der kartographierende Blick vieler Arbeiten der wissenschaftlichen Geographien der Technik durch den Wandel

\footnotetext{
${ }^{1}$ vgl. auch Internet-Angebote und Programme zur Signalwegkartierung wie „VisualRoute“ u.a., welche im Prinzip einen ähnlichen Repräsentationsschritt vollziehen.
} 
ihrer Gegenstände im Kern verändert wurde: Die abgebildete Technik und deren Artefakte mögen erstaunliche Innovationen erlebt haben - die geographische Annahme der Möglichkeit ihrer objektgleichen Verortung bleibt dagegen weitgehend intakt.

Damit sei diese Perspektive jedoch nicht pauschal als „Irrweg" kritisiert. Die Leistung dieses Zugriffs besteht gerade in der Überführung ihres Gegenstandes in eine diesem genau nicht inhärente, sondern ihm fremde Logik - die des gedanklichen oder manifesten Kartierens. Darin besteht zum einen der Mehrwert dieses Zugriffs - der ,fremde Blick“ des Kartographen wird dem Verständnis von Technik hinzugefügt. Aber genau diese Fremdheit ist es, die das Argument für eine hermeneutische, rekonstruktive Geographie des Technischen - neben dem kartierendem Zugang - so schlagend macht. Im Folgenden sollen theoretische Zugänge vorgestellt und diskutiert werden, die eine grundlegende Remodellierung des Phänomens des technischen Wandels in Bezug auf das Räumliche für sich beanspruchen.

\section{Modelle der Transformation soziotechnischer Raumverhältnisse in der Spätmoderne}

Die Humangeographie hat raumrelevante Technologien vor allem als Agenten des Ausgreifens der Lebensbezüge im $\mathrm{Zu}$ ge der Industrialisierung und Modernisierung verstanden. So hat Thrift (1996:42; mit Bezug auf Bradley, 1988:2f) die Reichweiten und biographischen Geographien verschiedener Generationen seit dem 19. Jahrhundert nachgezeichnet und damit die Moderne und ihre Geschichte als Expansionserzählung bzw. Expansionskartographie dargestellt. Während sich die Generation der Urgroßväter lediglich in einem ländlichen Nahraum bewegte, kommt die Großvatergeneration bereits mit großstädtischem Leben in Berührung. Der Vater hat darauf folgend eine auf das Nationale und auf das nahe Ausland reichende Mobilität erlangt, während der gegenwärtig lebende Sohn potientiell und real globale Lebensbezüge knüpft. Umgekehrt wird, in einem zweiten sehr bekannten Beispiel von Dicken (1998:152), die Ausweitung der Reichweiten im Zuge der Entwicklung von modernen Verkehrsmitteln (von Kutsche/Schiff über die Eisenbahn bis zum Flugzeug) als raumzeitliche Schrumpfung dargestellt, welche die Welt durch abnehmende Distanzwiderstände im Sinne von Erreichbarkeiten immer näher zusammenrücken lässt.

Was bei diesen Darstellungen im Fokus steht, sind historisch jeweils neue Möglichkeiten der körperlich-physischen Mobilität von Personen im Verlaufe der Moderne. Damit ist aber ein fast noch wichtigerer Teil der technischen Moderne nicht abgebildet: die Transformation von Räumlichkeit durch neue Kommunikationsmedien. Ein erster theoretischer $\mathrm{Zu}$ gang stellt Zeichen und Informationen dabei analog zu mobilen Körpern (Personen und Güter) vor. Die Wirkung und Gestalt von Technik würde dann ebenfalls im bekannten Abbildungsprinzip der Vergegenständlichung repräsentiert. Tech- nik wird wiederum als Mittel der Raumüberwindung und weniger als Mittel der Raumproduktion beschrieben - so jedenfalls der Eindruck, den die Globalisierungsdebatte zum Teil hinterlässt (vgl. Castells, 2000 [1996]; Cairncross, 1997; Albrow, 1996; zum Teil Appadurai, 2000).

Die eigentliche Hervorbringung des Räumlichen durch Technik wurde zunächst - wenn auch lediglich in allgemeiner Form - von der handlungszentrierten Sozialgeographie reflektiert (Werlen, 1999, 2007). Die idealtypische Unterscheidung zwischen traditionellen und spätmodernen Gesellschaften hat die Aufmerksamkeit auf umfassende Entankerungsprozesse gelenkt, welche über die physisch-körperliche Mobilität hinausreichen. Bewegt werden mit dem Einzug der Moderne außerdem Wissen, Informationen, Normen, Traditionen und soziale Positionen - unter anderem durch komplexe und leistungsfähige abstrakte Expertensysteme (Giddens, 1996:107ff; Werlen, 2010:31f). Das heißt, eine im Prinzip entankerte Lebenswelt der Spätmoderne ermöglicht den Subjekten neue Entscheidungen und Weltbezüge, verlangt ihnen aber diese Praktiken der „Welt-Bindung“ bzw. Wiederverankerung auch ab. Diese Weltbindung kann dann, dem Analyserahmen von Werlen folgend, z.B. durch ökonomische, normative oder symbolische Praktiken erfolgen, die einer je spezifischen Handlungslogik folgen. Das Technische wird hier handlungstheoretisch gleichzeitig zur Bedingung, zum Mittel und zum Ergebnis ,alltäglicher Regionalisierungen“ (Werlen, 1999, 2007).

Im angloamerikanischen humangeographischen Diskurs wird Technik vor allem in Form des Wandels, den die digitale Revolution auslöst, vielfältig thematisiert. Neue Geographien des Technischen bilden ein Kernthema der Debatte (stellv. Brunn et al., 2004; Brown und Laurier, 2005; Dixon und Whitehead, 2008; Graham, 1998, 2005; Thrift und French, 2002; Thrift, 2004; Dodge und Kitchin, 2005a; Kwan, 2007a) - sei es die kritische Analyse von Überwachungstechnik (s. z.B. Adey, 2004; Graham, 2005:572f), das Phänomen der technischen Strukturierung des Handelns durch Infrastrukturen am Beispiel des Flughafens (Adey, 2007), die lebensweltliche Erfahrung des mobilen Alltags (Laurier, 2004; Bissell, 2009) oder die Allgegenwart des digitalen Code (Dodge und Kitchin, 2005b). Digitale Techniken werden als konstitutiv für (neue) Erfahrungen und Affektmomente thematisiert - mit Bezug auf Videogames bei Shaw und Warf (2009), im Hinblick auf Geobrowsing von Kingsbury und Jones III. (2008) oder auch im Hinblick auf die emotionale Zuwendung zur Lebenswelt (Kwan, 2007b). Insbesondere neue soziale Ungleichheiten in Form der automatisierten Differenzierung von Nutzerklassen und asymmetrischer Zugangschancen (Graham, 2005:565ff) bilden einen wiederkehrenden Fluchtpunkt der Diskussion. Somit zeigt sich in der angloamerikanischen geographischen Debatte eine kultur- bzw. gesellschaftszentrierte und deutlich kritische Beleuchtung der vielfältigen „Mikrotransformationen“ und Machtbeziehungen (s. z.B. Graham et al., 2013), die mit dem Einzug digitaler Technik im Alltag verbunden sind. 
Durch die grundsätzlich hohe Aufmerksamkeit für das Thema werden zwar viele aktuelle empirische Phänomene aufgegriffen, es scheint dabei allerdings immer wieder ein statischer Raumbegriff durch, etwa wenn Technik im Sinne einer Invasion das Städtische durchdringt, wie wir es bei Thrift beschrieben finden:

...the geography of computing is changing shape. From being centred and stable entities located at definite sites, through the medium of wireless computing, computing is moving out to inhabit all parts of the environment and users are able to be mobile. (Thrift, 2004:182).

Das Argument überschreitet den bekannten Rahmen einer Mobilisierung und des räumlichen Ausgreifens im Kern kaum. Auch wenn mit Formeln wie der ,,automatic production of space“" (Thrift und French, 2002) und den ,transductive practises“ (Dodge und Kitchin, 2005a) theoretisch ein neuer handlungsbezogener, konstruierter Raum des Technischen angepeilt ist, so wird doch im Kern ein konventioneller Raumbegriff benutzt (die Figur Technik im Raum; Raum als Bühne und „Unterlage“ der Technikdiffusion), welcher für die Konstruktion der Invasionsmetapher unverzichtbar ist. Dies wird beispielsweise deutlich in Dodge und Kitchins (2011) Darstellung der Durchdringung des modernen Zuhauses (ebd., 159ff) oder der Orte des Konsums (ebd., 192) durch Software und ,coded objects“. Wird diese Form der Betrachtung dann konsequent fortgeführt, können diese Geographien in raumtheoretischer Hinsicht zumeist lediglich negativ formulierte Grobbefunde erzeugen. Es wird oft recht pauschal die Heterogenität und die Auflösung überkommener Raumbindungen festgestellt:

...they [die Räume der Software, T.F.] are inherently multitudinous, diverse and ambivalent, and operate at multiple scales... (Graham, 2005:565).

...the boundaries between the computational, spatial, social, and environmental dimensions are increasingly blurred. Each is entwined and reflected in the others in many interesting and subtle ways (Sui, 2004:67).

This means that the coded transduction of space is never fixed and shifts with place, time and context (Dogde und Kitchin, 2005a:174).

The representation of places has no ontological security $[. .$.$] the geospatial content enacted in aug-$ mented reality is necessarily spatially, temporally, and personally context-dependent (Graham et al., 2013:467).

Mit derlei Aussagen werden gängige Denkmuster der Postmoderne paraphrasiert - diese lassen sich aber auch als theoretisch-begriffliches Defizit interpretieren. Ein Raumverständnis, welches lediglich die zitierten Schlussfolgerungen zulässt, hat keine echte Erklärungskraft mehr. Ein Unterscheiden als Mindestanforderung an einen Begriff ist kaum mehr möglich. Die empirische Allgegenwart und die Ubiquität von technischen Objekten führt letztlich lediglich zu Negativbefunden, in dem man benennt, was nicht mehr gilt, welche Raumbindungen durch Technik aufgelöst werden bzw. welche neuen Bindungen überall und damit nirgends entstehen $^{2}$.

So ermöglicht die Fortschreibung eines im Kern statischen Raumbegriffes, welcher das Technische lediglich als räumlich Flüchtiges benennbar macht, zwar das Aufzeigen wesentlicher historischer Transformationen (im Vergleich mit dem Vorzustand), verliert aber aktuell deutlich an Beschreibungs- und Erklärungskraft. Im Wesentlichen fällt die Technikgeographie immer wieder in das Muster ,Technisierung = Raumdurchdringung/Raumsuspendierung“ zurück. Um hier eine neue Perspektive zu gewinnen, soll im Folgenden eine phänomenologische Betrachtung des Technischen diskutiert und daraus ein Verständnis des Technikgebrauchs als Interaktionshandeln entwickelt werden. Die zu untermauernde These wäre, dass eine erneuerte Handlungsund Sinnorientierung im Stile der interpretativen (Wissens)Soziologie das skizzierte raumbegriffliche Problem beheben helfen kann.

\section{Geographien und Phänomenologien der Digitalisierung}

Die dargestellten geographischen Zugänge haben entweder das Technische als Rauminhalt erforscht (s. Horvath, 1974 u.a., Abschnitt 2), oder aber sie haben das Technische in Form von Mitteln der Mobilität und Kommunikation als Kennzeichen der Moderne thematisiert. Entankerung und Globalisierung wären die entsprechenden Stichworte. Was hierbei noch nicht hinreichend beschrieben und erfasst ist, sind die lebensweltlichen Begegnungsmomente mit dem Technischen, welches sich allgemein als ,verschränkte“ Gesellschaft (Latour, 2006:36), als ,,society made durable“ (Latour, 1991) verstehen lässt. Dabei ist weniger die Frage leitend, welches theoretische Modell das Technische am besten erfasst (ob als Organersatz/Organverstärkung für das Mängelwesen Mensch (Gehlen, 2007 [1957]:5ff), nichtmenschliche Aktanten bzw. „Hybride“ (Latour, 1995:7ff) oder gar „Cyborgs“ (Haraway, 1995:33)), sondern wie Technik dem „Lebensweltbewohner“ begegnet. Wie erscheint das Technische innerhalb individueller und sozialer Praktiken? Wie erfolgt alltagsweltlich der Zugang zu Technik? Wie wird im Umgang und in der Interaktion mit Technik Geographie ge-

\footnotetext{
${ }^{2}$ Als Indiz für dieses Problem kann auch die intensive Rezeption des Begriffes ,everyware“ gelten (Greenfield, 2006, zit. v. Dodge und Kitchin, 2011:215ff), womit die Allgegenwart und/oder die beliebige Mobilität des Code gemeint ist.
} 
macht? Es geht also um die Spezifizierung eines phänomenologischen und wissenssoziologischen Denkens.

In der Spätmoderne hat Technik durch Arbeitsteilung, Rationalisierung und Spezialisierung einen Komplexitätsgrad erreicht, der sie in aller Regel dem Laiennutzer in ihrer Binnenstruktur unzugänglich macht. Abstrakte Expertensysteme (Giddens, 1996:107ff) kennzeichnen als Formen einer „distributed agency“ (Rammert, 2007:8) unseren Alltag. Diese Systeme treten als „Black Box“ (vgl. Callon, 2006 [1980]:56) ebenso allgegenwärtig auf wie sie dem Laien undurchsichtig erscheinen. Aus diesem Grundbefund leitet sich auch ein Großteil der Technikkritik des 20. Jahrhunderts ab, indem beobachtet wird, dass die Entlastungswirkung von Technik in zunehmendem Maße immer auch ein Entfremdungsmoment enthält (Gehlen, 2007 [1957]:23ff, 47ff). Am einfachen Beispiel der Türklingel hat der Philosoph Hans Blumenberg diese Begegnung mit dem Technischen reflektiert, indem er die Technikentwicklung als ein Verschwinden der Unmittelbarkeit in ihrem Gebrauch beschreibt:

Ich wähle das primitive Beispiel einer Türklingel. Da gibt es die alten mechanischen Modelle von Zugklingeln oder Drehklingeln: betätigt man sie, so hat man noch das unmittelbare Gefühl, den beabsichtigten Effekt in seiner Spezifität zu erzeugen, denn zwischen der tätigen Hand und dem erklingenden Ton besteht ein adäquater Nexus, d.h. wenn ich vor einer solchen Einrichtung stehe, weiß ich nicht nur, was ich tun muss, sondern auch, weshalb ich es tun muss. Anders bei der elektrischen Klingel, die durch einen Druckknopf betätigt wird: die Verrichtung der Hand ist dem Effekt ganz unspezifisch und heteromorph zugeordnet - wir erzeugen den Effekt nicht mehr, sondern lösen ihn nur noch aus. Der gewünschte Effekt liegt apparativ sozusagen fertig für uns bereit, ja er verbirgt sich in seiner Bedingtheit und in der Kompliziertheit seines Zustandekommens sorgfältig vor uns, um sich uns als das mühelos Verfügbare zu suggerieren“ (Blumenberg, 1981:35).

Diese Gleichzeitigkeit von Intransparenz und alltäglicher Zuhandenheit lässt sich auf das grundsätzliche Auseinanderdriften von Experten- und Laienhandeln zurückführen (vgl. Hartmann, 2012:261ff). Die leistungsfähigste, fortgeschrittenste Technik unseres Zeitalters, die digitale Hochtechnologie, bezieht ihren Erfolg ja gerade daraus, dass sie extrem abstrakte und komplexe Operationen (Expertenseite) als äußerlich einfachen Output-Effekt, als simpel abrufbare Leistung, in unserem Alltag verfügbar macht (Seite des Laiennutzers). Sie bietet fertige Sets von Ereignissen, deren automatisierte Verknüpfung, an (vgl. Schulz-Schaeffer, 1999). Wir Laiennutzer sind lediglich mit dem „Befehl-Erteilen“ und der Output-Verarbeitung betraut. Von der Perspektive der lebensweltlichen Erfahrung her betrachtet, verbirgt diese Technik ihre komplexe Struktur. Dass es sich um digitale Technik handelt, die sich im Kern stets auf einen binären Code zurückführen ließe, wird dem Nutzer genau nicht vermittelt. Die denkbar große Kluft zwischen der maximalen Abstraktheit des digitalen Algorithmus ${ }^{3}$ und der zunehmend einfachen Zugänglichkeit - meist in Form eines graphischen Interface - wird für den Nutzer unsichtbar. In dieser phänomenologischen Hinsicht ist deshalb die Rede von der digitalen Revolution alles andere als selbstverständlich. Ihren Erfolg verdankt diese Technik aktuell nämlich genau dem Umstand, dass ihre digital konstituierte Binnenstruktur nahezu vollständig unsichtbar und nicht erfahrbar wird, sie vielmehr immer perfektere äußere Formen ,artifizieller Präsenz“ (Wiesing, 2005) erzeugt. Aus handlungstheoretischer Sicht ließe sich ihr Siegeszug damit begründen, dass sie dem Nutzer genau keine revolutionären Transformationen seines Handelns und Erfahrens abverlangt, sondern im Gegenteil immer intuitiver, das heißt, der äußeren Erscheinung nach ,nichttechnischer" in unseren Alltag tritt. Es zeigt sich das Paradox einer ,emulierten Einfachheit“ und „,künstlichen Unmittelbarkeit", die jedoch auf komplexe Weise erzeugt wird. Technik reduziert sich zunehmend auf „Interfaces“ und „Screens“ - wir interagieren mit ihr symbolisch und visuell, ohne uns auf ihren Eigencharakter und ihre Binnenstruktur einlassen zu müssen. Das kann man als Triumph oder Entmündigung des Laien verstehen - es bleibt aber der Grundbefund, den eine Geographie des Technischen ernst nehmen sollte.

Methodische und theoretische Hinweise für die Berücksichtigung des Begegnungs- und Interaktionsaspekts finden sich bereits in den so genannten Workplace Studies (Heath und Luff, 2000; zu workplace geographies: Crang, 1994; McMorran, 2012), teilweise in den Science und Technology Studies (STS, s. stellv.: Latour, 2006; Furlong, 2010), in der geographisch orientierten Medienwissenschaft (Döring und Thielmann, 2009; Günzel, 2010; Abend und Thielmann, 2011) und in der kommunikationsorientierten Humangeographie (s. u.a. Laurier und Brown, 2008; Kingsbury und Jones III., 2008; Shaw und Warf, 2009; Haklay, 2010; Adams, 2010). Auch der Diskurs um eine neue handlungs- und interaktionsorientierte Kartographie bzw. Geomediennutzung gibt hierzu Anstöße (stellv. Elwood, 2006; Goodchild, 2007; Elwood et al., 2011; Bittner et al., 2013). Raum wird in den genannten Forschungsbereichen einerseits als Setting der Interaktion mit Technik untersucht, und/oder es wird nach den symbolischen Interaktionsinhalten (verbale und andere Zeichen) gefragt. Was in den Mittelpunkt rücken soll, ist die Fokussierung des Räumlichen innerhalb der Interaktionsinhalte. Ganz grundlegend gesprochen, wären Zeichen zu identifizieren und zu deuten, deren Gehalt für einen gelingenden

\footnotetext{
${ }^{3}$ Dabei zeigt sich, dass digitale Technik ein hervorragendes Beispiel liefert für die Wittgensteinsche These, nach der sich die Bedeutung eines Zeichens nur im Zeichengebrauch konstituiert und nicht durch ein durch das Zeichen bezeichnetes Objekt bestimmt wird. Denn im Falle des digitalen Algorithmus spiegelt das Zeichen nicht nur eine Aktion - es ist selbst die Operation/Aktion.
} 
Gebrauch von Technik maßgeblich ist und die ihrer Bedeutung nach einen Raum konstruieren bzw. die beanspruchen, auf Raum zu verweisen. Von der Interaktion im Raum wäre also - analog zum Wechsel der geographischen Forschungsperspektive auf Technik - zum (symbolischen) Raum in der Interaktion überzugehen.

\section{Alltäglicher Technikgebrauch als Interaktionshandeln und Geographie-Machen}

Die Interaktionssituation des alltäglichen Gebrauchs von Technik zeichnet sich, wie gezeigt wurde, durch die beiden Positionen des abstrakten Expertensystems einerseits und des Laiennutzers andererseits aus ${ }^{4}$. Das Interaktionshandeln kann demnach schematisch verstanden werden als eine Übersetzungsoperation zwischen den Zeichen, welche das System intern für sein Funktionieren konstruiert und den Zeichen, welche die Alltagssprache für den jeweiligen konkreten Kontext des Technikgebrauchs vorhält. Selbstverständlich geschieht diese Übersetzung nicht reibungs- und restlos, nicht vollständig und automatisch, denn das würde bedeuten, dass Technik sich vollkommen widerstandsfrei in unser Alltagshandeln integrierte, ja, das Technik - phänomenologisch - verschwinden würde. Sie erforderte dann weder Aufmerksamkeit noch irgendeine vom Laien erbrachte Verstehensleistung. Real zeigt sich dagegen aktuell oft noch das Bild, dass sich Zeichen, die einem System- und Expertenkontext entstammen, im Interaktionszusammenhang mit lebensweltlichen und alltagssprachlichen Zeichen abwechseln und dass beide Formen - wenn auch nicht immer systematisch und nachvollziehbar - aufeinander bezogen sind. Deduktiv ließen sich modellhaft drei Dimensionen des raumbezogenen Interaktionsinhalts unterscheiden, die jeweils durch unterschiedliche Praktiken gestützt werden:

- die Aneignung technischer, raumbezogener Zeichen durch den Laien

- die Emulation lebensweltlicher, subjekt- bzw. körperzentrierter Raumbegriffe durch die Sprache und Symbolik des Interface

- der Gebrauch allgemeinverständlicher Toponyme als kontextübergreifende Möglichkeit zur Adressierung des Räumlichen

Die erste Form, das Erlernen technischer Raumsymbolisierungen, bildet einen überkommenen Bestandteil der Sozialisierung in modernen Gesellschaften: Viele der komplexen

\footnotetext{
${ }^{4}$ Der Dualismus der Situation von System und Nutzer mag zwar ontologisch in vielfache, netzwerkartige Verknüpfungen und Richtungen einzuordnen bzw. auflösbar sein (wie im Rahmen der Akteur-Netzwerk-Theorie; s. z.B. Belliger und Krieger, 2006). Phänomenologisch bleibt er aber evident, weil der Laiennutzer im Moment der Interaktion mit technischen Systemen im Allgemeinen kaum Kenntnisse über diese komplexen Vernetzungen besitzt.
}

Infrastrukturen (Transport, Mobilität, Versorgung, Kommunikation) fordern vom Laien, dass er die systemische Raumkodierung wenigstens in Ansätzen nachvollzieht. Erst dann wird diese Technik in routinehafter Form nutzbar. So müssen wir etwa Postleitzahlen, ortsspezifische Telefonvorwahlnummern, die Fahrtliniennummern öffentlicher Verkehrsmittel oder auch die Kodierung von DVDs nach Weltregionen kennen, um mit dem jeweiligen technischen System erfolgreich interagieren zu können. Die Verarbeitung orts- und objektbezogener Informationen erfolgt zumeist in numerischer Form. Und weil - wie im Falle traditionell gestalteter Benutzeroberflächen - noch keine automatisierte Übersetzung in alltagssprachlich leichter verständliche Raumzeichen durch das Interface stattfindet, bleiben dem Laiennutzer diese numerischen Raumkodierungen ${ }^{5}$ nicht verborgen, sondern im Gegenteil wird deren Erlernen zur Vorbedingung der Nutzung. Kognitiv mag der überwiegend numerisch repräsentierte Raumbezug zwar zunächst nicht leicht zu verarbeiten sein - ein Hinterfragen seitens des Nutzers bleibt bei einem sich zuverlässig einstellendem Handlungserfolg aber zumeist aus.

Im Falle des Gebrauchs aktueller digitaler Technik ist nun der Erwerb von Kenntnissen über die numerische „Binnenkodierung" der Black Box - etwa das Erlernen von Programmiersprachen oder auch GIS-ähnlicher Elemente des Systems - durch die Konstruktion mehr oder minder nutzerfreundlich gestalteter Interfaces nicht mehr zwingend erforderlich. Über umfassende Systemkenntnisse verfügt neben den Bereitstellern und Konstrukteuren lediglich eine neue, selbstbewusste, gestaltende Gruppe von „Expertennutzern“. Sie beschränkt ihre Interaktion nicht auf die zum Output-Abruf notwendigen praktischen Routinen, sondern erfasst und verändert das bereitgestellte System ,im Inneren“. Quantitativ ist dieser Nutzertyp aber nicht dominant. Entsprechend der so genannten 90-9-1-Regel (Nielsen, 2006; Neis und Zipf, 2012:152 ${ }^{6}$ ) lässt sich vermuten, dass $90 \%$ der Nutzer sich auf den Output beschränken, lediglich $9 \%$ die Systemfunktion verstehen und nur $1 \%$ diese nicht nur versteht, sondern auch verändert. Wir müssten die aktuell herrschende Interface-Kultur insgesamt interpretieren als Schritt, den Nutzer von systeminternen Zusammenhängen und systemspezifischen Raumsymbolisierungen zu entlasten. Als Übersetzer der numerischen in allgemeinverständliche Raumzeichen fungiert nicht mehr der Nutzer, sondern - in automatisierter Weise - das Interface.

Diese Entlastung entspricht der zweiten der drei oben angeführten Interaktionsdimensionen. Ersetzt werden die numerischen Raumzeichen des Systems durch graphische Raumvisualisierungen und lebensweltbezogene deiktische

\footnotetext{
${ }^{5}$ Diese Kodierungen sind Ausdruck allgemeiner Merkmale technischer Fachsprachen wie Ökonomie, Eindeutigkeit, das Metapherntabu und ihre Anonymität (Roelcke, 1999:60ff).

${ }^{6}$ Zur Frage der Partizipationsungleichheit konnten beispielsweise Neis und Zipf (2012) zeigen, dass lediglich $5 \%$ der Nutzer von Open Street Map $90 \%$ des Inhalts erstellen.
} 
Ausdrücke. Neue Geomedien etwa bieten nicht mehr den „Blick von nirgendwo“ (Nagel, 1992) der klassischen Kartographie, sondern konstruieren egozentrierte Räume, welche digitale Rauminformationen automatisch in körperbezogene, deiktische (zeigende) und indexikalische (situationsgebundene) Raumzeichen übersetzen: hier, dort, rechts, links, neben, etc. Der „Pfeil“ eines Navigationssystems steht paradigmatisch für diese automatisierte Produktion/Emulation von Indexikalität. Schon der perspektivische Raumblick des Interface entlastet den Nutzer davon, sich in die klassischmoderne, letztlich abstrakte Idee eines universalen Erdraumes hineindenken zu müssen. Der ursprünglich selbst zu leistende Transfer zwischen Subjekt- und Erdraum wird dem Laiennutzer des Geomediums kaum mehr abverlangt. Theoretisch können diese neuen Kommunikations- und Konstruktionsformen als digitale Produktion des ,,place“ (s. z.B. Tuan, 1976:268ff; Buttimer, 1976:283ff) verstanden werden - als Konstruktion des subjektiv angeeigneten und lebensweltlich relevanten Raumes bzw. Ortes.

In diesem Wechselspiel aus Aneignungspraktiken des Laien und Emulationsleistungen des Interface ist die gesellschaftliche „Rahmung“ des Interaktionsgeschehens aber noch nicht berücksichtigt. Die Alltagssprache, mit der sich die Subjekte verständigen, besteht nicht nur aus den flüchtigen, situations- und körpergebundenen Raumzeichen der Deixis. Moderne Gesellschaften haben einen Komplexitätsgrad und schlicht eine Größe erreicht, welche die Raumbezeichnung auch unabhängig von konkreten einzelnen Interaktionssituationen möglich machen muss. Es müssen auch je aktuell abwesende Räume, Orte und Objekte symbolisiert werden können. Schon die komplexe Konstruktion des Nationalstaates macht diese Notwendigkeit unmittelbar einsichtig. Daraus folgt für das Problem des Interaktionshandelns mit Technik, dass mit dieser dritten, gesellschaftlich konstruierten Geographie eine Zeichenklasse hinzukommt, die gewissermaßen universal, das heißt, kontextübergreifend nutzbar ist. Diese Raumzeichen sind Toponyme - konkrete oder abstrakte Ortsnamen und Regionsbezeichnungen. Sie stehen für kollektiv geteilte Weisen der Raumbezugnahme bzw. Raumkonstruktion, an die sowohl der Experte wie auch der Laie ,,andocken“ können, um eine Verständigung im Sinne gelingender Interaktion mit Technik zu erreichen. So bildet die „Nationalisierung“ des Internets ein Beispiel, wie sich allgemeine gesellschaftliche Geographien im Interaktionsinhalt abbilden: Web-Adressen mit Nationenkürzel, die nationenbezogene Segmentierung des Marktes im E-Commerce (z.B. „Amazon“) oder auch die länderkundliche Gestaltung von Tourismusseiten sind Indizien dafür. Fasst man den Bereich des Interaktionshandelns mit Technik so weit, dass er auch den alltäglichen Medienkonsum mit einschließt, wird diese dritte Form der Raumsymbolisierung sogar noch deutlicher sichtbar. Und zwar nicht nur deren integrative Rolle, sondern auch ihr widersprüchlicher Charakter. So werden etwa regionale Identitäten über potentiell globalisierte und digitalisierte Medien stabilisiert. Es entsteht ein Span- nungsverhältnis, in dem technisch kontingente Praktiken der Informationsvermittlung vermeintlich inkontingente Identitäten und Ortsbezüge konstruieren helfen. Dabei erfolgt eine unsichtbare Kopplung abstrakter technischer, algorithmischer Raumkodierungen mit Ideen traditioneller bis primordialer Verankerung. Die ,,vorgestellten Gemeinschaften“ (Anderson, 1996) der Nation oder Region bemühen zu ihrer Stabilisierung technische Funktionszusammenhänge, die den nationalen territorialen Rahmen regelmäßig überschreiten. Diese Überschreitungen müssen für eine kohärente inhaltliche Vorstellung von Nation oder Region vom Medienkonsumenten notwendig ausgeblendet werden. In Anlehnung an Ernest Renans Hinweis, dass die Mitglieder einer Nation vieles vergessen haben müssen, um sich mit der Idee der Nation identifizieren zu können (Renan, 1996 [1882]:14), kann man mit Blick auf das digitale Zeitalter schlussfolgern, dass die Mitglieder der Nation über die technischen Geographien wenig bis gar nichts erfahren sollten, um die Idee der Nation aufrecht erhalten zu können und diese kohärent erscheinen zu lassen.

So erscheint für diese dritte Ebene der Raumsymbolisierung die These naheliegend, dass die Abstraktheit und Intransparenz digitaler Medien und Infrastrukturen (z.B. Smartphones, Geobrowser, Navigationssysteme) genau nicht die Aneignung universaler und potentiell globaler Raumdeutungsmuster fördert, sondern deren Gegenteil. Werden nämlich technische Zusammenhänge der Raumkonstruktion tendenziell undurchsichtig und verschwinden genuin technische, z.B. numerische, Raumzeichen aus dem Interaktionszusammenhang $^{7}$ mit dem Ziel der Nutzerentlastung, dann ist die mediale und technische Konstruktion des „Urwüchsigen“, „Verankerten“, „Natürlichen“, ,Ewigen“, anscheinend sogar immer widerspruchsfreier möglich. Je weniger wir im Alltag mit ,rohen“ technischen Raumkodierungen konfrontiert werden, desto plausibler erscheinen uns traditionelle Container- und Verortungslogiken. Paradoxerweise wird so das Nichttechnisierte, etwa die subjektive Sehnsucht nach Ortsbindung oder die vermeintlich naturräumliche Fixierung des Kollektivs, mit technischen Mitteln alltäglich realisiert. Der Widerspruch besteht dabei aber nicht, wie oft behauptet wird, im Auseinanderklaffen von materieller Praxis einerseits und inadäquater symbolischer Repräsentation dieser Praxis andererseits in dem Sinne, dass wir in einer materiell entankerten/globalisierten Welt leben, dies aber nicht in unseren Weltbildern angemessen spiegeln. Stattdessen besteht das Problem darin, dass eine reale, technisch-symbolische Raumkonstruktion nicht (mehr) wahrgenommen wird, weil sie phänomenologisch gesehen in der „Black Box“ verschwindet. Dagegen lässt sich das weiße, leere Blatt, als das man sich das nahezu beliebig gestaltbare Interface digita-

\footnotetext{
${ }^{7}$ Für diesen Befund sprechen die skizzierten Formen der Emulation lebensweltlicher und kollektiver Raumbegriffe durch immer „intuitiver“ zu handhabende Raumkonstruktionen des InterfaceDesign.
} 
ler Medien vorstellen kann, zunehmend fast ohne technische Zwänge mit allen möglichen, vor allem aber mit den ,gängigen" Raumkonstruktionen (z.B. Region, Landschaft, Nation etc.) füllen. Technische Gestaltungszwänge schwinden und machen überkommenen Raumkonzepten Platz, vermutlich auch, weil diese dem Nutzer wenig Lernaktivität abverlangen.

Unabhängig von diesen kritischen Erwägungen bleibt die analytische Unterscheidung numerischer (systembezogen), deiktischer (nutzerbezogen) und toponymischer (kontext/gesellschaftsbezogen) Raumzeichen als vorläufiger Analyserahmen anwendbar. Diese deduktive Einteilung lässt sich vorbehaltlich ihrer empirischen Korrektur und Erweiterung damit erklären, dass die geographische Paradigmenkonstellation ähnliche Raumkonzepte und deren Unterscheidung nahelegt. So hat die raumwissenschaftliche Geographie mit der Quantifizierung und Objektivierung des Raumes eine Vorleistung für die technisch-mediale Geographie des Alltags erbracht. Die „Humanistic Geography“ hat mit körper- und erfahrungsbezogenen Geographien vorweggenommen, was man im Interaktionszusammenhang aktuell als Nutzer- und Egozentrierung beobachten kann. Und die konstruktivistische Sozialgeographie hat mit der kritischen Rekonstruktion länderkundlicher und regionalgeographischer Raumkonzepte die Rahmung, die Betrachtung des gesellschaftlichen Kontextes, in dem alltäglicher Technikgebrauch stattfindet, ermöglicht. Gerade wenn man berücksichtigt, dass wissenschaftliche Ideen in die Alltagswelt zurückfließen (,,doppelte Hermeneutik“, Giddens, 1995:429f), kann man erwarten, dass die von wissenschaftlicher Seite bereits intensiv reflektierten Raumkonzepte auch einen Teil des alltäglichen, symbolischen Interagierens mit Technik bilden. Wenn sich hier auch nicht eindeutige Rezeptionspfade nachweisen lassen, ergeben sich doch mindestens deutliche Parallelen zwischen wissenschaftlicher und alltäglicher Raumkonstruktion. In diesem Sinne sind die Konzepte und Raumdeutungsangebote der wissenschaftlichen Geographie methodisch für die Untersuchung alltäglichen Geographie-Machens instrumentalisierbar - sie werden als methodische Werkzeuge selbst zu Technik.

Geht man also davon aus, dass vorläufig mindestens drei grundsätzlich verschiedene Wege der Raumkonstruktion im Interaktionshandeln verknüpft werden, wird die alltägliche Routine des Technikgebrauchs als Übersetzungsoperation verstehbar. Aus dieser Perspektive heraus bedeutet Technikgebrauch nicht mehr primär die gelingende Nutzung materieller Mittel im Sinne der Objekttransformation, der Organverstärkung bzw. Organersatzes (Gehlen), sondern Technikgebrauch schließt die bewusste oder routinenhafte Übersetzung einer symbolischen Raumkonstruktionsform in eine andere ein. Kompetenz des Laien im Umgang mit Technik ist in sozialgeographischer Hinsicht das Vermögen, routinenhaft verschiedene symbolische Geographien, d.h. Konstruktionsweisen des Räumlichen, handlungsseitig zu verketten. Dies zeigt sich im grundsätzlichen Verhältnis von potentiell globalisierten digitalisierten Medien und ihren regionalisierenden Inhalten ebenso wie etwa im alltäglichen Gebrauch von Infrastrukturen der Mobilität. Zum Beispiel verlangt ein banaler Fahrscheinkauf am Automaten je nach Gestaltungsform der Benutzeroberfläche von der Nutzerin, die numerische Zielortkodierung, ihr eigenes körperliches Hier und die Namen von Abfahrts- und Zielort aufeinander beziehen zu können. Dass solche Fähigkeiten zum routinehaften Gebrauch gesellschaftlich relativ weit verbreitet sind, sollte den Blick darauf nicht verstellen, dass dennoch für viele Nutzer die Interaktion mit Technik eine Herausforderung, das Überwinden einer Schwelle darstellt, die sich unter anderem aus den komplexen und abstrakten Raumkodierungen technischer Systeme erklärt.

\section{Fazit}

Am Beginn der Betrachtung stand das Ziel, Technik nicht mehr primär objekthaft im Raum zu untersuchen, weil dadurch - so die These - tendenziell die Sinn- und Bedeutungsebene des Technischen vernachlässigt wird. Auch wäre die Fortschreibung des Primates des Materiellen und Erdräumlichen zu umgehen, wie sie in Zeitdiagnosen der beschleunigten Raumüberwindung bzw. Raumvernichtung angelegt ist. Es gilt, nicht den Ort und Raum des Technischen zu suchen, sondern die Raum konstruierenden Aspekte des Technikgebrauchs in den Blick zu nehmen. Die Grundidee des Textes bildete die Hinwendung der Geographie der Technik zum Interaktionshandeln. Die Interaktion zwischen einem abstrakten System und einem Laiennutzer bildet modellhaft die Grundkonstellation alltäglichen Technikgebrauchs. Handeln mit Technik wird aus dieser Perspektive als eine im Kern sprachliche Fähigkeit verstanden - als eine Fähigkeit, verschiedene Raumsymbolisierungen aufeinander beziehen und diese ineinander übersetzen zu können. Dazu wurde vorläufig ein deduktiv-hypothetisches Schema dreier bekannter Raumkonzepte erörtert, die im Technikgebrauch ,,veralltäglicht" werden: numerische, deiktische und toponymische Raumsymbolisierungen. Eine Geographie alltäglichen Technikgebrauchs sollte die Sensibilität für die Herausforderungen dieser Übersetzungsoperation entwickeln. Sie sollte zu verstehen suchen, wie hier Schwellenmomente und Problemsituationen in Alltagsroutinen aufgelöst werden. Im Bereich Mobilität etwa könnte so der Fokus auf die Herausforderungen der Interaktion mit einer komplexen Infrastruktur gelegt werden, indem man nach den alltäglichen Praktiken des Verstehens von deren symbolischer Geographie durch den Nutzer fragt. Dies könnte die Geographie der Mobilität erweitern, indem Mobilität nicht primär „Körpertransport“ von A nach B meint oder vornehmlich nach extrinsischen Mobilitätsanlässen gefragt würde.

Aus der Anwendung der hier skizzierten Perspektive ergeben sich außerdem zwei systematische Problemfelder, die in empirischen Studien genauer untersucht werden könnten. 
Das erste Feld geht von dem skizzierten Befund aus, dass sich digitale Technik, wie sie zunehmend unseren Alltag prägt, dem Nutzer immer weniger in ihrer Binnenstruktur offenbart. Sie zieht sich gewissermaßen immer stärker in die „Black Box“ zurück. Gleichzeitig aber, und dies macht den Erfolg und Fortschritt dieser Technik aus, wird den Gestaltern des Interface immer mehr freie Hand gelassen. Das Interface kann modular relativ unabhängig von den internen Funktionszusammenhängen designt werden - nicht mittels technisch-numerischer Raumkodierungen muss kommuniziert werden, sondern es eröffnen sich theoretisch völlig neue Möglichkeiten, Raum zu konstruieren. Durch diese neu gewonnene Freiheit von technischen Zwängen bietet sich aber eben auch die Möglichkeit des Rückgriffs auf Raumkonzepte, welche dem Nutzer lange vertraut sind. Traditionelle Raumkonzepte können die Interaktion erleichtern, weil sie dem Laiennutzer bekannt sind - unabhängig von technischen Kenntnissen. So könnte - und dies wäre eine zu prüfende These - die neue Freiheit im Interface-Design (also ironischerweise der technische Fortschritt) zu dem realen Effekt führen, dass lange bekannte Formen der Konstruktion von Orten, Landschaften und Ländern verstärkt Eingang in die System-Nutzer-Interaktion finden. Denn diese Formen werden nun immer seltener durch technische Geographien unmittelbar irritiert und erschüttert, wie es für die klassische Moderne der Industrialisierung noch typisch war $^{8}$. Das zweite Problemfeld zeigt sich, wenn man nach Ausnahmen vom Grundbefund der „Black Box“ und des sich immer lebensweltferner abspielenden technisch-digitalen Operierens Ausschau hält. Gibt es tiefer gehende Gegenbewegungen zur technischen Differenzierung und zur wachsenden Intransparenz von Technik insgesamt? Wo wird real am Grundbefund der „Black Box“ gerüttelt? Dies geschieht nicht nur im Falle der bereits erwähnten Nutzerexperten und Prosumer, die an der Konstruktion eines technischen Systems mitwirken, anstatt sich auf die Outputkonsumtion zu beschränken. Vergleichbares könnte man auch beobachten in Tendenzen der Retraditionalisierung im Bereich der Warenproduktion und Konsumtion. Die Kenntnisse um den Produktionszusammenhang bilden ein immer wichtigeres Kaufargument für den Laien - der Wunsch nach der Durchdringung des Schleiers über dem Bereitstellungszusammenhang vieler Produkte (Lebensmittel, Textilien u.a.), welcher im Verlaufe der Moderne immer undurchschaubarer wurde, ist nunmehr deutlich vernehmbar. Autarke Gemeinschaften von Selbstversorgern (Teilen statt Tauschen) wären das vielleicht extremste Beispiel einer Reaktion auf die Entfremdung von

\footnotetext{
${ }^{8}$ Wahrscheinlich haben - historisch verglichen mit der Digitalisierung des Alltags - die Eisenbahn oder das fordistische Produktionsregime deutlich radikaler die Vorstellungen und Materialitäten der Menschen und Landschaften umgekrempelt. Geobrowser oder die Augmented Reality App des Smartphone jedenfalls fügen sich doch erstaunlich nahtlos an unsere vertrauten Raumvorstellungen an. Sie irritieren und revolutionieren nicht, sondern bestätigen viel mehr bekannte Konzepte.
}

technischen Produktionszusammenhängen. Diese Tendenzen sind nicht mit schierer Technikkritik oder pauschaler Technikskepsis zu verwechseln. Sie sind eher Ausdruck der Skepsis gegenüber dem Grundprinzip, auf die Aneignung technischen Expertenwissens zu verzichten zugunsten der Entlastungseffekte, die durch Technik eintreten.

Edited by: B. Korf

Reviewed by: two anonymous referees

\section{Literatur}

Abend, P. und Thielmann, T.: Die Erde als Interface. Ein GoogleEarth-Rundgang, in: Raum als Interface. Massenmedien und Kommunikation, Herausgeber: Mitcherich, A., 187/188, 127143, 2011.

Adams, P.: A taxanomy for communication geography, Prog. Hum. Geog., 35, 37-57, 2010.

Adey, P.: Surveillance at the airport: Surveilling mobility/mobilizing surveillance, Environ. Plann. A, 26, 1365-1380, 2004.

Adey, P.: 'May I have your attention': airport geographies of spectatorship, position, and (im)mobility, Environ. Plann. D, 25, 515536, 2007.

Albrow, M.: The global age: state and society beyond modernity, Cambridge, Polity, 1996.

Anderson, B.: Die Erfindung der Nation. Zur Karriere eines folgenreichen Konzepts, Frankfurt/New York, Campus, 1996.

Appadurai, A.: Modernity at Large. Cultural Dimensions of Globalization, Minneapolis, University of Minnesota Press, 2000.

Belliger, A. und Krieger, C. (Hrsg.): ANThology. Ein einführendes Handbuch zur Akteur-Netzwerk-Theorie 2006, Bielefeld, Transcript, 2006.

Bissell, D.: Visualising everyday geographies: practises of vision through traveltime, T. I. Brit. Geogr., 34, 42-60, 2009.

Bittner, C., Glasze, G., und Turk, C.: Tracing contingencies: analyzing the political in assemblages of web 2.0 cartographies, GeoJournal, 78, 935-948, 2013.

Blumenberg, H.: Wirklichkeiten in denen wir leben, Stuttgart, Reclam, 1981.

Bradley, D. J.: The scope of travel medicine, in: Travel Medicine, Herausgeber: Steffen, R., Lobel, H. O., Haworth, J., und Bradley, D. J., Berlin, Springer, 1988.

Brown, B. und Laurier, E.: En-Spacing Technology: Some Thoughts On The Geographical Nature of Technology, in: Spaces, Spatiality and Technology, Herausgeber: Turner, P. und Davenport, E.: Dordrecht, Springer, 19-30, 2005.

Brunn, S., Cutter, S. und Harrington, J. W. (Hrsg.): Geography and Technology, Dordrecht/Boston/London, Kluwer, 2004.

Buttimer, A.: Grasping the dynamism of lifeworld, Ann. Assoc. Am. Geogr., 66, 277-292, 1976.

Cairncross, F.: The Death of Distance. How the Communications Revolution Is Changing Our Lives, Boston, Harvard Business School Press, 1997.

Callon, M.: Die Sozio-Logik der Übersetzung: Auseinandersetzungen und Verhandlungen zur Bestimmung von Problematischem und Unproblematischem, in: ANThology. Ein einführendes Handbuch zur Akteur-Netzwerk-Theorie, Herausgeber: Bel- 
liger, A. und Krieger, D., Bielefeld, Transcript, 51-74, 2006 [1980].

Castells, M.: The Rise of the Network Society, Cambridge, Mass., Blackwell, 2000 [1996].

Crang, P.: It's showtime: on the workplace geographies of display in a restaurant in southeast England, Environ. Plann. D, 12, 675704, 1994.

Dicken, P.: Global Shift. Transforming the world economy, London/Thousand Oaks/New Dehli, Sage, 1998.

Dixon, D. und Whitehead, M.: Technological trajectories: old and new dialogues in geography and technology studies, Soc. Cult. Geogr., 9, 601-611, 2008.

Dodge, M. und Kitchin, R.: Code and the Transduction of Space, Ann. Assoc. Am. Geogr., 95, 162-180, 2005a.

Dodge, M. und Kitchin, R.: Codes of life: identification codes and the machine readable world, Environ. Plann. D, 23, 851-881, 2005b.

Dodge, M. und Kitchin, R.: Code/space: software and everyday life, Cambridge, MIT Press, 2011.

Döring, J. und Thielmann, T. (Hrsg.): Mediengeographie. Theorie Analyse - Diskussion, Bielefeld, Transcript, 2009.

Elwood, S.: Negotiating Knowledge Production: The Everyday Inclusions, Exclusions, and Contradictions of Participatory GIS Research, The Professional Geographer 58, 197-208, 2006.

Elwood, S., Schuurman, N. und Wilson, M.: Critical GIS, in: The SAGE Handbook of GIS and Society, Herausgeber: Nyerges, T., Couclesis, H. und McMaster, R., Los Angeles u.a., Sage, 87-106, 2011.

Furlong, K.: Small technologies, big change: Rethinking infrastructure through STS and geography, Prog. Hum. Geog., 35, 460482, 2010.

Gehlen, A.: Die Seele im technischen Zeitalter, Frankfurt, Klostermann, 2007 [1957].

Giddens, A.: Die Konstitution der Gesellschaft, Frankfurt, Campus, 1995.

Giddens, A.: Konsequenzen der Moderne, Frankfurt, Suhrkamp, 1996.

Goodchild, M.: Citizens as sensors: the world of volunteered geography, GeoJournal, 69, 211-221, 2007.

Graham, M., Zook, M. und Boulton, A.: Augmented reality in urban places: contested content and the duplicity of code, T. I. Brit. Geogr., 38, 464-479, 2013.

Graham, S.: The End of geography or the explosion of space? Conceptualizing space, place and information technology, Prog. Hum. Geog., 22, 165-185, 1998.

Graham, S.: Software-sorted geographies, Prog. Hum. Geog., 29, 562-580, 2005.

Günzel, S.: Medialer Raum: Bilder - Zeichen - Cyberspace, in: Raum. Ein interdisziplinäres Handbuch, Herausgeber: Günzel, S. und Kümmerling, F., Metzler, Stuttgart, 219-233, 2010.

Haklay, M. (Hrsg.): Interacting with Geospatial Technologies, Oxford, Wiley \& Sons, 2010.

Haraway, D.: Die Neuerfindung der Natur, Primaten, Cyborgs und Frauen, Frankfurt, Campus, 1995.

Hartmann, F.: Symbiosis yet? Koevolution der Grenzfläche Mensch/Maschine, in: Visualität in der digitalen Medienkultur, Herausgeber: Freyermuth, G. und Gotto, L., Bildwerte, Bielefeld, Transcript, 259-285, 2012.
Heath, C. und Luff, P.: Technology in Action, Cambridge, Cambridge University Press, 2000.

Horvath, R.: Machine Space. The Geographical Review, 64, 166187, 1974.

Kingsbury, P. und Jones III., J. P.: Walter Benjamin's Dionysian Adventures on Google Earth, Geoforum, 40, 502-513, 2008.

Kwan, M.: Mobile Communications, Social Networks, and Urban Travel: Hypertext as a new Metaphor for Conceptualizing Spatial Interaction, The Professional Geographer, 59, 434-446, 2007a.

Kwan, M.: Affecting Geospatial Technologies - Toward a feminist politics of emotion, The Professional Geographer, 59, 22-34, $2007 b$.

Latour, B.: Technology is Society made durable, in: A Sociology of Monsters, Herausgeber: Law, J., London, Routledge, 103-131, 1991.

Latour, B.: Wir sind nie modern gewesen. Versuch einer symmetrischen Anthropologie, Berlin, Akademie Verlag, 1995.

Latour, B.: Ethnographie einer Hochtechnologie: Das Pariser Projekt „Aramis“ eines automatischen U-Bahn-Systems, in: Technografie. Zur Mikrosoziologie der Technik, Herausgeber: Rammert, W. und Schubert, C., Frankfurt, Campus, 25-60, 2006.

Laurier, E.: 'Doing office work on the motorway', Theor. Cult. Soc., 21, 261-277, 2004.

Laurier, E. und Brown, B.: Rotating maps and readers: praxiological aspects of alignment and orientation, T. I. Brit. Geogr., 33, 201216, 2008.

Licoppe, C., Diminescu, D., Smoreda, Z. und Ziemlicki, C.: Using mobile phonegeolocalisation for 'socio-geographical' analysis of co-ordination, urbanmobilities, and social integration patterns, Tijdschrift for Economische enSociale Geografie, 99, 584-601, 2008.

McMorran, C.: Practising workplace geographies: embodied labour as method in human geography, Area, 44, 489-495, 2012.

Nagel, T.: Der Blick von nirgendwo, Frankfurt, Suhrkamp, 1992.

Neis, P. und Zipf, A.: Analyzing the Contributor Activity of a Volunteered Geographic Information Project - The Case of Open Street Map, ISPRS International Journal of GeoInformation, 1, 146-165, 2012.

Nielsen, J.: Participation inequality: Encouraging more users to contribute, http://www.useit.com/alertbox/participation_ inequality.html (last access: 18 October 2012), 2006.

Rammert, W.: Technik - Handeln - Wissen, Zu einer pragmatistischen Technik- und Sozialtheorie, Wiesbaden, VS, 2007.

Renan, E.: Was ist eine Nation? Rede am 11. März 1882 an der Sorbonne, Hamburg, Europäische Verlagsanstalt, 1996 [1882].

Roelcke, T.: Fachsprachen, Berlin, Schmidt, 1999.

Rosenberg, S.: Dreaming in Code, New York, Random House, 2007.

Schulz-Schaeffer, I.: Technik und die Dualität von Ressourcen und Routinen, Z. Soziol., 28, 409-428, 1999.

Shaw, I. und Warf, B.: Worlds of affect: virtual geographies of video games, Environ. Plann. A, 41, 1332-1343, 2009.

Sui, D.: GIS, Cartography, and the "Third Culture": Geographic Imaginations in the Computer Age, The Professional Geographer, 56, 62-72, 2004.

Thrift, N.: Spatial Formations, London u.a., Sage, 1996.

Thrift, N.: Remembering the technological unconscious by foregrounding knowledges of position, Environ. Plann. D, 22, 175190, 2004. 
Thrift, N. und French, S.: The automatic production of space. T. I. Brit. Geogr., 27, 309-335, 2002.

Torrens, P. M.: 'Wi-Fi Geographies', Ann. Assoc. Am. Geogr., 98, 59-84, 2008

Tranos, E.: The Geography of the Internet. Cities, Regions, and Internet Infrastructure in Europe, Elgar Publishing, Cheltenham/Horthhampton, 2013.

Tuan, Y.: Humanistic Geography, Ann. Assoc. Am. Geogr., 66, 266-276, 1976.

Werlen, B.: Wolfgang Hartke - Begründer der sozialwissenschaftlichen Geographie, in: Sozialgeographie und Soziologie. Dialog der Disziplinen, Herausgeber: Heinritz, G. und Helbrecht, I., Münchner Geographische Hefte 78, Passau: L.I.S. Verlag, 15-41, 1998
Werlen, B.: Zur Ontologie von Gesellschaft und Raum, Sozialgeographie alltäglicher Regionalisierungen, Bd. 1, Stuttgart, Steiner, 1999.

Werlen, B.: Globalisierung, Region und Regionalisierung, Sozialgeographiealltäglicher Regionalisierungen, Bd. 2, Stuttgart, Steiner, 2007.

Werlen, B.: Gesellschaftliche Räumlichkeit 2: Konstruktion geographischer Wirklichkeiten, Stuttgart, Steiner, 2010.

Wiesing, L.: Artifizielle Präsenz, Frankfurt, Suhrkamp, 2005. 